\title{
BMJ Open Urgent assessment and ongoing care for infection in community-dwelling older people: a qualitative study of patient experience
}

\author{
Abigail Moore (D) , ${ }^{1}$ Sara McKelvie, ${ }^{1}$ Margaret Glogowska, ${ }^{1}$ Daniel S Lasserson, ${ }^{2}$ \\ Gail Hayward
}

To cite: Moore A, McKelvie S, Glogowska M, et al. Urgent assessment and ongoing care for infection in communitydwelling older people: a qualitative study of patient experience. BMJ Open 2021;11:e043541. doi:10.1136/ bmjopen-2020-043541

- Prepublication history and additional material for this paper is available online. To view these files, please visit the journal online (http://dx.doi.org/10. 1136/bmjopen-2020-043541).

Received 13 August 2020 Revised 03 February 2021 Accepted 26 February 2021

Check for updates

(C) Author(s) (or their employer(s)) 2021. Re-use permitted under CC BY-NC. No commercial re-use. See rights and permissions. Published by BMJ.

${ }^{1}$ Nuffield Department of Primary Care Health Sciences, University of Oxford, Oxford, UK

${ }^{2}$ University of Warwick Warwick Medical School, Coventry, UK

Correspondence to

Dr Abigail Moore;

abigail.moore@phc.ox.ac.uk

\section{ABSTRACT}

Objectives To explore the experience of infection from the perspective of community-dwelling older people, including access and preferences for place of care.

Design Qualitative interview study, carried out between March 2017 and August 2018.

Setting Ambulatory care units in 0xfordshire, UK. Participants Adults $>70$ years with a clinical diagnosis of infection.

Methods Semistructured interviews based on a flexible topic guide. Participants were given the option to be interviewed with their caregiver. Thematic analysis was facilitated by NVivo V.11.

Results Participants described encountering several barriers when accessing an urgent healthcare assessment which were hard to negotiate when they felt unwell. They valued home comforts and independence if they received care for their infection at home, though were worried about burdening their family. Most talked about hospital admission being a necessity in the context of more severe illness. Perceived advantages included monitoring, availability of treatments and investigations. However, some recognised that admission put them at risk of a hospital-acquired infection. Ambulatory care was felt to be convenient if local, but daily transport was challenging. Conclusions Providers may need to think about protocols and targeted advice that could improve access for older people to urgent healthcare when they feel unwell. General practitioners making decisions about place of care may need to better communicate risks associated with the available options and think about balancing convenience with facilities for care.

\section{INTRODUCTION}

Older people with infection are at high risk of unscheduled hospital admissions. ${ }^{1-3}$ However, hospital admission is associated with harms in this age group, such as physical deconditioning, delirium and hospital-acquired infection. ${ }^{4}$ There has been a recent focus in the UK to make health services work better for community-dwelling older people. Potential solutions include better identifying frailty, supporting independence, providing timely
Strengths and limitations of this study

- Qualitative methods have allowed in-depth exploration of the experiences of community-dwelling older people.

- There is an over-representation of ambulatory care as a management strategy for infection among our sample.

Our results may not be transferable to patients with infections of different severities.

access to healthcare, developing ambulatory care infrastructure and delivering care closer to patients' homes. ${ }^{5-9}$ Older people have identified the importance of autonomy and independence in relation to their care. ${ }^{510}$

In a previous qualitative study, UK general practitioners (GPs) said that their instinct was not to admit older people to hospital if possible, and they perceived that older people preferred to remain at home. GPs talked of weighing up the risks of admission to older people (including not mobilising, losing muscle mass, thromboembolic events, worsening confusion and hospitalacquired infections) compared with the risks of community-based care (including falls, deterioration and death). They liked to involve patients and their families in discussions about management, and used this shared decision making as way to reduce their personal liability. ${ }^{11}$

However, little is known about the experiences and preferences of older people regarding their treatment in the context of acute infection. Understanding their perspective is important to ensure that necessary service redesign is patient centred. ${ }^{12}$ Likewise, a broader understanding of patient experience could help primary care professionals when approaching complex decisions around place of care. 
This qualitative study aimed to explore the experience of receiving treatment for an infection from the perspective of community-dwelling older people and their caregivers, including access to an urgent assessment and preferences for place of care in this context.

\section{METHODS}

\section{Recruitment}

Older people aged 70 and over with a clinical diagnosis of infection and capacity to consent to an interview were recruited from three ambulatory care units in Oxfordshire. The ambulatory care units in Oxfordshire provide a same day medical assessment and, if necessary, follow-up for patients referred from primary care. Patients have potentially more severe illnesses that cannot be managed by the GP alone. The units can do blood tests,X-rays and provide intravenous treatments for patients but do not normally admit patients overnight. They provide a multidisciplinary team assessment and can add additional support for the patient to support them in their homes during their acute illness. Participants were ineligible if they could not communicate in English or could not participate in an interview within 2 months of their index visit to the ambulatory care unit.

Sampling was purposive, with an attempt to recruit people with a range of ages and infections and from all three units. Clinical team members approached potential participants face-to-face and consent was sought for the research team to contact them about the study. These potential participants were telephoned within 2 weeks of their clinical contact by researchers (AM and SM). They were given further information and, if they agreed to take part, an interview date set within a further 6 weeks. Participants were not paid to take part in the study.

Recruitment continued until the study team agreed that data saturation had been reached. We defined data saturation as no amendments to the topic guide, no new codes and no new significant themes for several consecutive interviews. ${ }^{13}$ In addition, we reached a point where we had sufficient detail to explain and elaborate on the themes which emerged from the analysis. ${ }^{13}$

\section{Data collection}

$\mathrm{AM}$, a female GP trainee and researcher, and SM, a female salaried GP and researcher, both trained in qualitative methods, conducted the interviews between March 2017 and August 2018. None of the participants were known to the researchers. All participants were given the option to invite a caregiver to join the interview. Interviews were carried out face to face in participants' homes. There was no-one else present in the interview besides the participant, caregiver (if invited) and the researcher. Participants were told the aims of the study and gave written informed consent prior to the interview.

Participants were asked to talk about their most recent experience of infection. Interviews were semistructured following a flexible topic guide developed and pilot tested by the research team (see online supplemental box 1). ${ }^{14}$ The topic guide was initially constructed with some a priori issues based on the existing literature, ${ }^{15} 16$ the team's clinical experience and a previous interview study with GPs. ${ }^{111718}$ While this oriented the two interviewers to broad areas around the experience of infection, the participants also had the opportunity to raise issues of their own, in their own words. As a result, the topic guide evolved during the study period following team discussions of emerging themes. ${ }^{19}$

Interviews lasted 40-60 min, were audiorecorded and transcribed verbatim by a professional transcriber.

\section{Patient and public involvement}

Neither patients nor public were involved in the design and conduct of this study.

\section{Data analysis}

A pragmatic approach to thematic analysis was used. ${ }^{19}$ Coding and analyses of the data were completed by two researchers (AM and SM) using NVivo (V.11) to support data management. The interview transcripts were open coded, with initial double coding to check for consistency between the two researchers. Related codes were combined create to categories. Material within the categories was subsequently reviewed for explanation, before looking at how the categories combined to support emerging narratives. Earlier interviews were recoded in the light of ongoing analysis and early analysis brought out topics which were added to the topic guide.

AM and SM established an audit trail from the raw data of the interview transcripts through coding to development of themes to ensure dependability. The research team discussed the initial coding framework, as well as ideas for categories emerging from the data, and subsequently, the final themes to ensure their credibility and confirmability. Disagreements were resolved by consensus. Group meetings were also used to reflect on personal experience and field notes to improve reflexivity.

\section{RESULTS}

Sample

Thirty-five eligible patients who were approached agreed to be contacted by the study team. Twenty-two patients consented to participate and were interviewed, 6 of them with a carer, making 28 participants altogether (online supplemental figure S1). Participant characteristics are summarised in table 1, with further details in online supplemental table S1.

\section{Findings}

Themes emerging from discussion with participants are summarised in table 2 and are explored in more detail below. 


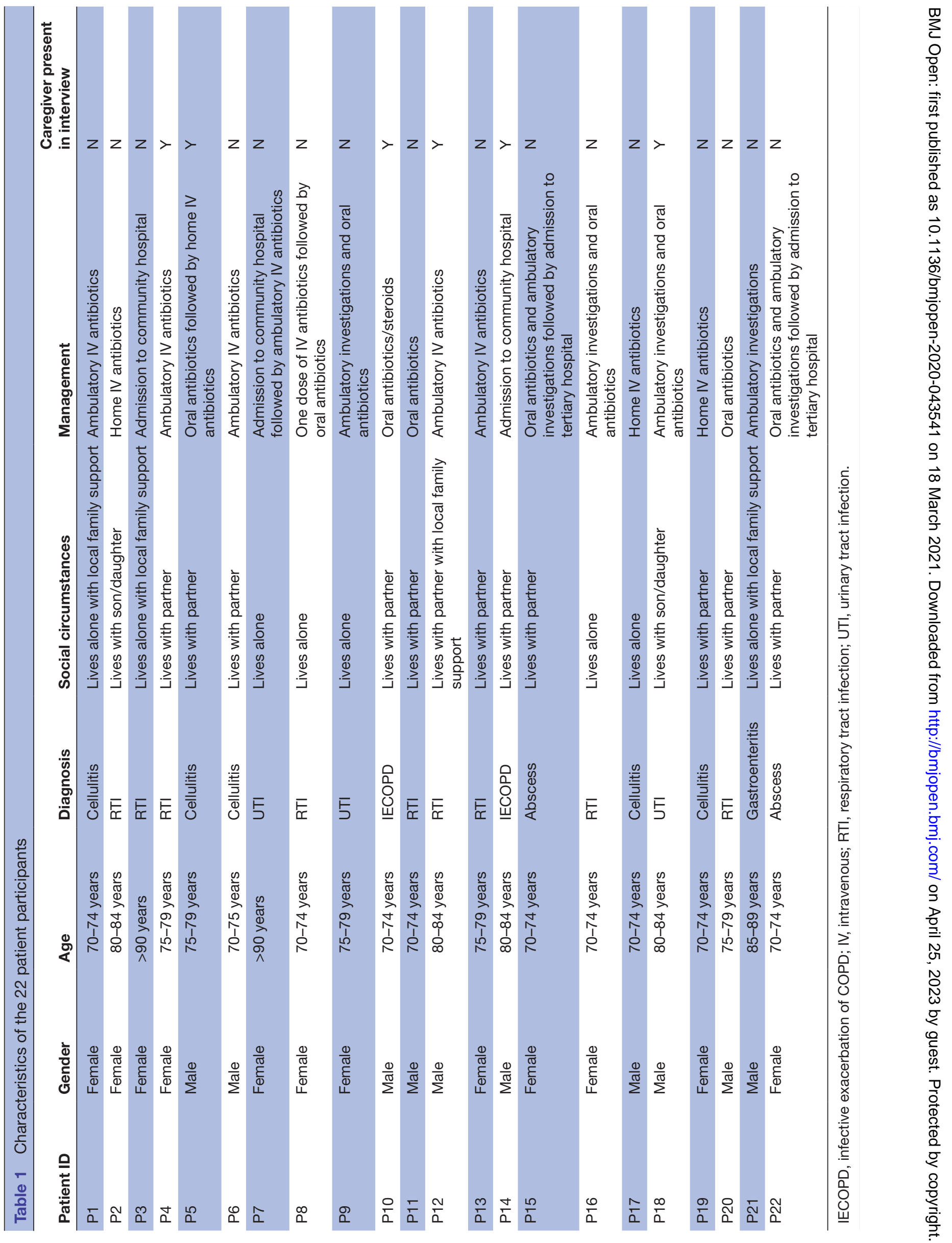


Table 2 Themes and subthemes emerging from discussion with patients about the preferences for care in the context of infection

\begin{tabular}{ll}
\hline Major themes & Subthemes \\
\hline $\begin{array}{l}\text { Having an urgent healthcare } \\
\text { assessment for infection }\end{array}$ & $\begin{array}{l}\text { Access to an assessment } \\
\text { Experience of urgent } \\
\text { assessment }\end{array}$ \\
$\begin{array}{l}\text { Ongoing care for an } \\
\text { infection }\end{array}$ & $\begin{array}{l}\text { Care in the home } \\
\text { Hospitalisation } \\
\text { Ambulatory care }\end{array}$ \\
\hline
\end{tabular}

Theme 1: having an urgent healthcare assessment for infection Access to an assessment

Most participants talked about negotiating a number of barriers to access an urgent healthcare assessment. For many, the first was an automated telephone service at the GP practice or 111. Participants perceived that these services involved confusing options or a long wait to get through to a person, sometimes resulting in them giving up.

You've got to get through, first of all, five different options from some robot before you can get through to anybody. P19 (female, 70-74 years, cellulitis), regarding the GP practice

Once through to a person on the phone, participants talked about having to articulate their problem and what they wanted to happen next. Some participants found that GP reception staff were very accommodating and did not question a request for same day assistance.

I phoned the receptionist and I said, 'Please can you ask doctor to come and see me because I'm not well.' [um] She said, 'Yes, of course,' she said... Now I know that if I said, 'I need him to phone me before surgery,' he would have done, you know, if I'd, if I'd pushed it.' P16 (female, 70-74 years, chest infection), regarding the GP practice

Others, however, found that reception staff could either be obstructive, or could put the onus back on the patient to determine the severity of their illness. Some participants said that they could be assertive and were able to express their needs when this happened; this was usually related to previous experience of negotiating the system, familiarly with the symptoms or having been educated or carefully safety-netted by their GP in the past.

If I'm, if I'm bad, and it's not getting any better, I phone up the GP and they say, 'Is it important, [name]?' and I say, 'I only phone up when it's important.' P20 (male, 75-79 years, RTI), regarding GP receptionists

In contrast, others said that they weren't confident when challenged by a receptionist and did not have enough knowledge to know what to say.
Well, Ijust said, 'Could I make an appointment with my doctor?' You know, there was the usual going through the list. 'Oh, well we've got one in three weeks.' ... 'I'm really struggling at the moment.' ... 'Well, you know, I'm afraid we haven't got one.' ... 'Can I have an emergency one?'... 'No, not unless it's really serious.' You know, it's that kind of, and at that point, I wasn't thinking it was serious. I was thinking, 'I've got a sore toe' P15 (female, 70-74 years, abscess), regarding GP receptionists

If not directly offered an urgent face to face assessment by 111 call handlers or a GP receptionist, participants described being triaged over the phone by a GP. Some participants felt there was a low threshold for them to be seen. Others described meeting resistance or again being asked to assess the severity of their illness. A minority of the participants got the sense that GPs were work averse.

The feeling is that they don't want to come out and so they'll do what they can do to sort of cover themselves without inconvenience, sorry it sounds awful, without inconveniencing themselves. P19 (female, 70-74 years, cellulitis)

Overall, the task of accessing same day assessment was described by many as an ordeal, requiring patience, perseverance and in some cases some insistence from the patient. Several felt that they needed an advocate to fight for this on their behalf, particularly as being unwell meant they had reduced energy or ability to express themselves clearly.

...you're trying to make your symptoms clear and your medical history very briefly clear, so that they can form some clinical judgement to what's happening and you're not feeling at all yourself and so, it's quite, it's quite a challenge. P19 (female, 70-74 years, cellulitis), regarding the GP practice

\section{Experience of urgent assessment}

Most participants were seen face to face by a GP as part of their initial assessment. Many participants talked of a special relationship with one GP at their practice who they preferred to see if possible. One reason for this preference was that their usual GP would know that they were actually unwell if they had chosen to seek care urgently. That said, most participants recognised that in the context of acute illness they may not be able to see their usual doctor and did not usually mind this.

If visiting their GP practice, several participants remarked that they were surprised about how quiet the practice was or how quickly they were seen. Several participants had preconceptions of general practice based on what they had seen or read in the media.

What amazed me when I got there. The surgery was, there was only about three people in it and after seeing all the hype on television I expected it to be 
packed with people coughing all over you. P10 (male, 70-74 years, IECOPD)

Several participants were assessed at home by their GP and tended to express surprise and/or gratitude for a visit. Other participants described how the availability of home visits was reduced compared with the past.

Most participants were happy with their assessment by the GP and the decisions that they made. Only one participant did not have confidence in the GP, and this was based on her extensive previous experience with a similar infection.

So, I felt that the first doctor I spoke to yesterday didn't want to visit, but she did want to do something, so she prescribed oral antibiotics and I knew in my heart that they wouldn't make any difference. But I couldn't say to her at that stage, I didn't know her. I couldn't say to her, 'No, that's not enough.' P19 (female, 70-74 years, cellulitis)

Two participants were seen at their GP practice by a healthcare professional who was trained in urgent assessment but was not a doctor. In contrast to the participants who were seen by GPs, they were not satisfied with their assessment and said they would have preferred to have seen a doctor.

So, in hindsight, you know, I think maybe I should have seen a doctor rather than a paramedic. Maybe a doctor would have then said, 'Right, I'm not going to send you home with a paracetamol. I'm going to send you up to the [tertiary hospital].' P22 (female, 70-74 years, abscess), about seeing a paramedic

Several participants were assessed by an out-of-hours $(\mathrm{OOH})$ GP. Most felt that this was suboptimal compared with seeing a GP at their own practice. One participant who was seen face to face by an OOH GP described them doing their best given the circumstances.

\section{Theme 2: ongoing care for an infection}

During interviews, participants were asked to reflect on how and where their care was managed with their recent infection. Participants were also asked to discuss hypothetical scenarios in which their care could have been delivered in an alternative way. Participants were asked for their perceptions of the advantages and disadvantages of different places of care. To answer this, the participants sometimes drew on their own previous experiences or experiences of friends and family.

Many participants found expressing views on place of care challenging. Several participants talked of their trust in doctors in making the right choice and not feeling qualified to express an alternative view about place of care.

I take the view that I'm not medically qualified. If a doctor is saying, "I'm admitting you to hospital," that is what is going to happen. I've no reason to sort of over challenge professional advice from a doctor because I'm not, I'm not professionally qualified to do so anyway. P18 (80-84 years, male, UTI)

\section{Care in the home}

Many participants described the positives of retaining their normal life if they received the majority of their care for their infection at home. Participants valued home comforts including access to their own things, home-cooked food and sleeping in their own bed and felt these added to a quality of life that they would not get in hospital.

You can wear what you like, you read what you want. You've got television for when you want it. It's just something that you have your life and then people come in and do the medical bit. P19 (female, 70-74 years, cellulitis)

Independence and mobility were also cited as advantages to remaining at home. This contrasted to the perceived restricted environment of being a hospital inpatient (see Hospitalisation, below).

Also I've been able to get a bit of fresh air, go out in the garden and do a little bit of weeding from standing position...if I'd have been in hospital I would have been taking up a bed, being miserable through not being able to get out and about and not getting the exercise... P5 (male, 75-79 years, cellulitis)

However, several participants said that they would have struggled to self-care at home when they were feeling unwell or would have needed to rely heavily on others. Some participants were worried about burdening their family or giving them responsibility for their health if they deteriorated at home.

Here if I'd suddenly felt really bad I would have people panicking and rushing me, wondering whether I should go in or out again. That didn't occur, but you can see that that would be quite a worry for other people if you were really poorly in here rather than in hospital. P13 (female, 75-79 years, RTI)

Participants who had experience of input from a home visiting team for their recent infection (eg, home intravenous antibiotics or dressing changes) described how it combined the benefits of home comforts with specialist treatment and follow-up. Most found it a convenient service that avoided travel and could be fitted around their life. Participants also valued the social aspect of regular contact and preferred that it made their treatments seem less medicalised by being located at home.

I could almost always say, 'It's great fun.' We turn this into a field hospital. We hang a coat hanger from the curtain rail. That is our little hanger for the drip and I sit here and while the drip is going through they do the observations and we have a chat and it takes about forty minutes, so it's just a question of using the time. P19 (female, 70-74 years, cellulitis) 
A few participants mentioned the downsides of a visiting service including the unpredictability of timing of visits, and the physical pain associated with regular intravenous cannulation.

\section{Hospitalisation}

Most participants talked about hospital admission being a necessity in the context of more severe illness rather than based on a personal request or preference.

...you can't have the necessary professional care if you're not in that environment. So, it's just something you, it's a calculated risk I suppose, and the doctors decide whether it's necessary or not. Female caregiver of P14 (80-84 years, IECOPD)

Most participants said they would not mind being in hospital for an infection, particularly if only for a short time. Many participants talked about their sense of an improved quality of the management that they could receive in hospital compared with the community. Facilities, investigations and treatment options were all felt to be better in hospital. Some perceived that this would result in an increased speed of recovery or conferred a survival advantage.

One advantage raised by participants was being monitored continuously by healthcare professionals during a hospital stay. This gave them a sense of security which they felt they would not get from the sort of informal checks they would get at home from a family member or caregiver (see Care in the home, above).

The advantages are the fact that you've, you've got twenty-four hour care, even though they wake you up at twelve o'clock at night to put you back on a drip. But yes, so you're being monitored. P22 (female, 7074 years, abscess)

Some participants talked about the convenience of having assistance with day to day tasks when they were not feeling their best, including having their meals provided and being given their medication. This was felt to be particularly important if they usually lived alone, where being in the hospital environment could also provide social interactions.

So, from the fact that you're fed and watered, that's good. The fact that you're giving your medication is also good because I do think that could be a big issue with the number of pills that I was taking or put on and I think for, you know, more elderly person, it's a little bit nice to be a little bit secure, you're in the right place at the right time. P15 (female, 70-74 years, abscess)

However, for others this closely controlled environment was felt to be too restrictive or boring, with one participant describing how her days in hospital were largely dictated by medical tasks.

It's almost like suspended animation. You're regulated by mealtimes, by getting up, by going to bed with the dispensation of the medicines and the observations and dressing your legs. P19 (female, 70-74 years, cellulitis)

The most commonly perceived downside of hospital admission was the risk of acquiring another infection and fears about the level of cleanliness; something that was felt to hold more significance for the older age group. Participants were also concerned about the burden of visiting on the family, especially if the hospital was further away from their home.

I was a bit worried if I had to go to the [tertiary hospital] or something. The time to get there, if [partner] wanted to come up to see me, you know, we're both fairly busy, where are you going to park, how long are you going to take? P14 (male, 80-84 years, IECOPD)

Some participants expressed a dislike of hospitals influenced by previous negative experience of a family member usually due to a perceived lack of care by staff.

\section{Ambulatory care}

All participants attended ambulatory care, located in hospital or standalone community sites, as part of the assessment/treatment of their recent infection. In particular, participants valued kindness and compassion from those treating them in this setting. Being offered refreshments was a commonly given as an example.

And little touches like you get there and they offer you a cup of coffee. That, that, it's only a small thing but it means a lot. P4 (female, 75-79, chest infection)

Community ambulatory care units were also felt by participants to be calmer and more organised than a hospital, and several commented on the speed of being seen, although there were exceptions to this. Participants generally felt that that staff had more time for them as an individual than in a hospital setting and that they were therefore more thorough in their assessments.

For some participants, the community ambulatory care units were much closer to them than the tertiary hospital, and they valued the accessibility of a local service.

But because it was so easy to get there and easy to park and you knew you were going to a place that was very friendly and kind of caring, it was fine. P4 (female, 75-79 years, chest infection)

However, the most commonly perceived criticism was the need for daily travel to and from ambulatory care units. Participants found that transport was timeconsuming and could place a considerable burden on friends or family. Participants who had to use organised transport described the frustrations of waiting around and the unreliability of this service.

You have an appointment at a clinic at ten o'clock in the morning, you had to be ready to be picked up at seven o'clock. I had to get up and get myself prepared for that and at times I sat here waiting, sitting 
here waiting with coat on and could sit for an hour, could sit for two hours before the ambulance came and by that time I'd missed my appointment. P7 (female, $>90$ years, UTI), regarding daily travel to an ambulatory care unit

\section{DISCUSSION}

\section{Summary}

Participants described encountering a number of barriers to accessing an urgent healthcare assessment: getting through on the phone, negotiating call handlers, articulating their problem and, in some cases, being asked to self-assess the urgency of their problem. Having to negotiate a same day assessment was described as being more difficult when feeling unwell.

Many participants described the positives of retaining their normal life if they received the majority of their care for their infection at home. Participants valued familiarity, home comforts and independence, though some participants were worried about burdening their family or failing to self-care adequately. Most participants talked about hospital admission being a necessity in the context of more severe illness. Perceived advantages included monitoring, availability of treatments and investigations as well as support with nutrition and medications. However, some recognised that admission could be restrictive and put them at increased of an additional hospital-acquired infection. Access to ambulatory care was felt to be convenient if available locally, but daily transport was challenging.

\section{Comparison with existing literature}

Some of the challenges associated with contacting UK primary care and getting an appointment have been described previously, particularly the frustrations of getting through to a GP practice on the telephone and uncertainty of what problems require a same-day assessment. ${ }^{20}$ Conversation analysis has shown that there can be a burden on patients to drive forward calls to receptionists to achieve service. ${ }^{21}$ To our knowledge, however, this is the first study to describe the added barriers for older people, and how experiencing an acute infection affects this process for them.

Older people have previously described hospital admissions as being unavoidable in the context of acute ill health $^{22}$ echoing our participants' views that hospital admission was sometimes a necessity. Whereas GPs said that their instinct was not to admit to hospital and they liked making shared decisions with patients about place of care, ${ }^{11}$ older people in this study said that they would not mind a hospital admission if a GP felt they needed it, and did not necessarily feel qualified to participate in the decision-making process.

Older people in this study also described a number of advantages of hospital not previously mentioned by GPs,${ }^{11}$ including the convenience of having assistance with everyday tasks, the security of regular monitoring and reduced burden and worry for family/caregivers compared with staying at home. These views are echoed in several existing qualitative studies exploring older patients views of a hospital stay in other European countries $^{2324}$ and in the response of a survey of older patients in the USA. ${ }^{25}$

The main risk of an admission identified by older people in this study was that of a hospital acquired infection. GPs considered there to be many more risks associated with a hospital stay, including not mobilising, losing muscle mass, thromboembolic events and worsening confusion. $^{11}$

\section{Strengths and limitations}

Qualitative methods have allowed in-depth exploration of the experiences of older people, a usually underrepresented group in research. ${ }^{26}$

There are some limitations of our sample when considering the transferability of our results. In recruiting all our participants from ambulatory care settings, we have an over-representation of this as a management strategy for infection, which may have influenced perceptions of both admission to hospital and staying at home. By design none of the participants had a significant cognitive impairment as they needed to have capacity to consent to the interview. We also recruited more people at the younger end of the older age spectrum, probably because younger people with fewer comorbidities were more likely to agree to participate in research. Despite these limitations, the views of people with more advanced age, with multimorbidity and with polypharmacy are captured among the sample (see table 1) and some participants and their caregivers referenced their other experiences of infection during their interviews including milder and more serious illnesses.

The interviews were conducted with the patients and their caregivers together creating a shared account of the recent infection and of related issues, where discrepancies didn't always emerge. We could not analyse the carers' views separately given the small numbers and the cocreated accounts.

Finally, both interviewers are GPs, which may have influenced how participants responded to some prompts relating to care from their own GP. However, our data demonstrate that participants felt able to be honest about their experiences including being critical of their GP practice.

\section{Implications for research and practice}

Standard gatekeeping to urgent primary care may be extra challenging for older patients. Reception training and protocols may need to reflect this and safety netting advice to older patients could include approaches to circumventing issues. Explicit advice about what would necessitate an urgent appointment may give older people confidence when requesting one in the future. 
GPs and older people do not necessarily consider the same risks and benefits to hospital admission for infection, and in general the perceptions of older people regarding hospital stays are not always as negative as GPs think. There is scope for further education of GPs to help them better understand older patient priorities, as well as education for older people in helping them appreciate the wider risks of a hospital admission and the local or community-based alternatives available. This would help communication in the shared decision-making process.

\section{CONCLUSIONS}

Older people can find negotiating gatekeepers to urgent primary care challenging, especially in the context of acute infection and providers may need to think about protocols, training and targeted advice that can improve this. GPs making decisions about place of care for older people with infection may need to improve the information they provide to patients about the associated risks of different options and think about balancing convenience with facilities for care.

Acknowledgements We thank the staff at the Ambulatory Assessment Unit (John Radcliffe Hospital, Oxford) and the Emergency Multidisciplinary Units (Abingdon and Witney) for their help with recruitment, and all the patients and caregivers who took part in the study.

Contributors AM, SM, MG, DSL and GH conceived and designed the study. AM and SM conducted the interviews. AM and SM coded the data and conducted the analysis, with regular meetings with MG, DSL and GH to discuss the coding framework, categories and themes. AM completed the first draft of the manuscript. $\mathrm{AM}, \mathrm{SM}, \mathrm{MG}, \mathrm{DSL}$ and GH contributed to revising the manuscript and gave final approval of the version published. AM, SM, MG, DSL and GH agreed to be accountable for all aspects of the work.

Funding This work was supported by the Oxfordshire Health Services Research Committee. SM is funded by the National Institute for Health Research (NIHR) Collaboration for Leadership in Applied Health Research and Care 0xford at Oxford Health NHS Foundation Trust. AM is funded by an NIHR Academic Clinical Fellowship. GH, MG and DSL are funded by the NIHR Community Healthcare MedTech and IVD Cooperative.

Disclaimer The views expressed are those of the authors and not necessarily those of the NHS, the NIHR or the Department of Health and Social Care.

Competing interests None declared.

Patient consent for publication Not required.

Ethics approval The study received ethical approval by the London - Fulham Research Ethics Committee (Reference: 16/L0/1902).

Provenance and peer review Not commissioned; externally peer reviewed.

Data availability statement No data are available. To protect the identity of the participants the audio files and full transcripts are not available.

Supplemental material This content has been supplied by the author(s). It has not been vetted by BMJ Publishing Group Limited (BMJ) and may not have been peer-reviewed. Any opinions or recommendations discussed are solely those of the author(s) and are not endorsed by BMJ. BMJ disclaims all liability and responsibility arising from any reliance placed on the content. Where the content includes any translated material, BMJ does not warrant the accuracy and reliability of the translations (including but not limited to local regulations, clinical guidelines, terminology, drug names and drug dosages), and is not responsible for any error and/or omissions arising from translation and adaptation or otherwise.

Open access This is an open access article distributed in accordance with the Creative Commons Attribution Non Commercial (CC BY-NC 4.0) license, which permits others to distribute, remix, adapt, build upon this work non-commercially, and license their derivative works on different terms, provided the original work is properly cited, appropriate credit is given, any changes made indicated, and the use is non-commercial. See: http://creativecommons.org/licenses/by-nc/4.0/.

\section{ORCID iDs}

Abigail Moore http://orcid.org/0000-0001-9546-2942

Gail Hayward http://orcid.org/0000-0003-0852-627X

\section{REFERENCES}

1 Quan TP, Fawcett NJ, Wrightson JM, et al. Increasing burden of community-acquired pneumonia leading to hospitalisation, 19982014. Thorax 2016;71:535-42.

2 Blunt I. QualityWatch: focus on preventable admissions. trends in emergency admissions for ambulatory care sensitive conditions, 2001 to 2013, 2013. Available: https://www.health.org.uk/sites/ default/files/QualityWatch_FocusOnPreventableAdmissions.pdf [Accessed 25 Jun 2020].

3 Wittenberg R, Sharpin L, McCormick B. Understanding emergency hospital admissions of older people, Centre for Health Service Economics \& Organisation, 2014. Available: https://www.chseo.org. uk/downloads/report6-emergencyadmissions.pdf [Accessed 25 Jun 2020].

4 Caplan GA, Sulaiman NS, Mangin DA, et al. A meta-analysis of "hospital in the home". Med J Aust 2012;197:512-9.

5 Oliver D, Foot C, Humphries R. Making our health and care systems fit for an ageing population King's Fund, 2014. Available: https:// www.kingsfund.org.uk/sites/default/files/field/field_publication file/making-health-care-systems-fit-ageing-population-oliver-foothumphries-mar14.pdf [Accessed 25 Jun 2020].

6 Carson D, Clay H, Stern R. Urgent care: a practical guide to transforming same-day care in general practice primary care Foundation, 2009. Available: https://www.primarycarefoundation.co. uk/images/PrimaryCareFoundation/Downloading_Reports/Reports_ and_Articles/Urgent_Care_Centres/Urgent_Care_May_09.pdf [Accessed 25 Jun 2020].

7 Future Hospital Commission. Future Hospital: caring for medical patients, 2013. Available: https://www.rcplondon.ac.uk/projects/ outputs/future-hospital-commission [Accessed 25 Jun 2020].

8 Monitor. Moving healthcare closer to home: literature review of the clinical impacts, 2015. Available: https://www.gov.uk/government/ uploads/system/uploads/attachment_data/file/459268/Moving healthcare_closer_to_home_clinical_review.pdf [Accessed 25 Jun 2020].

9 Age UK. Improving later life. services for older people - what works, 2014. Available: https://www.ageuk.org.uk/Documents/EN-GB/Forprofessionals/Research/Services-what_works_spreads.pdf?dtrk=true [Accessed 25 Jun 2020].

10 van de Pol MHJ, Fluit CRMG, Lagro J, et al. Quality care provision for older people: an interview study with patients and primary healthcare professionals. Br J Gen Pract 2015;65:e500-7.

11 Moore A, Croxson C, McKelvie S, et al. General practitioners' attitudes and decision making regarding admission for older adults with infection: a UK qualitative interview study. Fam Pract 2019;36:493-500.

12 The King's Fund. From vision to action: making patient-centred care a reality, 2012. Available: https://www.kingsfund.org.uk/sites/default/ files/field/field_publication_file/Richmond-group-from-vision-toaction-april-2012-1.pdf [Accessed 01 Feb 21].

13 Malterud K, Siersma VD, Guassora AD. Sample size in qualitative interview studies: guided by information power. Qual Health Res 2016;26:1753-60.

14 Edwards R, Holland J. What is qualitative Intervewing? London: Bloomsbury, 2013.

15 Utens CMA, van Schayck OCP, Goossens LMA, et al. Informal caregiver strain, preference and satisfaction in hospital-at-home and usual hospital care for COPD exacerbations: results of a randomised controlled trial. Int J Nurs Stud 2014;51:1093-102.

16 Kendall M, Murray SA, Carduff E, et al. Use of multiperspective qualitative interviews to understand patients' and carers' beliefs, experiences, and needs. BMJ 2009;339:b4122.

17 Hayward GN, Moore A, Mckelvie S, et al. Antibiotic prescribing for the older adult: beliefs and practices in primary care. $J$ Antimicrob Chemother 2019;74:791-7.

18 McKelvie S, Moore A, Croxson C, et al. Challenges and strategies for general practitioners diagnosing serious infections in older adults: a UK qualitative interview study. BMC Fam Pract 2019;20:56.

19 Ritchie J, Lewis J, McNaughton Nicholls C. Qualitative research practice. Second ed. London: Sage, 2014. 
20 McAllister F, Blunt A, Davies C. Research into accessing primary care services delivered in a general practice setting: a qualitative research study, 2019. Available: https://gov.wales/accessingprimary-care-services-qualitative-research [Accessed 01 Feb 2021].

21 Stokoe E, Sikveland RO, Symonds J. Calling the GP surgery: patient burden, patient satisfaction, and implications for training. $\mathrm{Br} \mathrm{J} \mathrm{Gen}$ Pract 2016;66:e779-85.

22 Themessl-Huber M, Hubbard G, Munro P. Frail older people's experiences and use of health and social care services. J Nurs Manag 2007;15:222-9.
23 van der Kluit MJ, Dijkstra GJ, de Rooij SE. The decision-making process for unplanned admission to hospital unveiled in hospitalised older adults: a qualitative study. BMC Geriatr 2018;18:318.

24 Hallgren J, Ernsth Bravell M, Dahl Aslan AK, et al. In hospital we trust: experiences of older peoples' decision to seek hospital care. Geriatr Nurs 2015;36:306-11.

25 Fried TR, van Doorn C, O'Leary JR, et al. Older person's preferences for home vs hospital care in the treatment of acute illness. Arch Intern Med 2000;160:1501-6.

26 Briggs R, Robinson S, O'Neill D. Ageism and clinical research. Ir Med J 2012;105:311-2. 